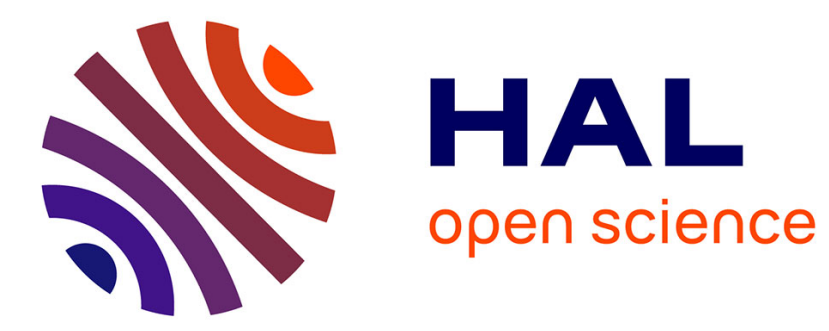

\title{
Simulating a random vector conditionally to a subvariety: a generic dichotomous approach
}

Frédéric Dambreville

\section{To cite this version:}

Frédéric Dambreville. Simulating a random vector conditionally to a subvariety: a generic dichotomous approach. SIMULTECH 2021, Jul 2021, Paris, France. hal-03164754v2

\section{HAL Id: hal-03164754 \\ https://hal.science/hal-03164754v2}

Submitted on 15 Mar 2021 (v2), last revised 14 Dec 2021 (v3)

HAL is a multi-disciplinary open access archive for the deposit and dissemination of scientific research documents, whether they are published or not. The documents may come from teaching and research institutions in France or abroad, or from public or private research centers.
L'archive ouverte pluridisciplinaire $\mathbf{H A L}$, est destinée au dépôt et à la diffusion de documents scientifiques de niveau recherche, publiés ou non, émanant des établissements d'enseignement et de recherche français ou étrangers, des laboratoires publics ou privés. 


\title{
Simulating a random vector conditionally to a subvariety: a generic dichotomous approach
}

\author{
Frédéric Dambreville ${ }^{1} \mathbb{1 D}^{\mathrm{a}}$ \\ ${ }^{1}$ Office national d'études et de recherches aérospatiales, 6 Chemin de la Vauve aux Granges, 91120 Palaiseau, France
}

Keywords: Rare event simulation, subvariety, interval analysis, black-box optimization

\begin{abstract}
The problem of sampling a random vector conditionnaly to a subvariety within a box (actually, a small volume around the subvariety) is addressed. The approach is generic, in the sense that the subvariety may be defined by an isosurface related to any (computable) continuous function. Our approach is based on a dichotomous method. As a result, the sampling process is straightforward, accurate and avoids the use of MCMC methods. Our implementation relies on the evaluation of the matching with the subvariety at each dichotomy step. By using interval analysis techniques for evaluating the matching, our method has been applied up to the dimension 11. Perspectives are evoked for improving the sampling efficiency on higher dimensions. A concept and an example of application of this simulation technique to black-box function optimization are detailed.
\end{abstract}

\section{INTRODUCTION}

The paper addresses the issue of sampling a given law defined on a $n$-dimension box conditionally to a subvariety of this box. Given a random vector $\boldsymbol{X}$ of density $f_{X}$ defined on $\mathbb{R}^{n}$, a box:

$$
[\mathbf{b}] \triangleq \prod_{k=1}^{n}\left[b_{k}^{-}, b_{k}^{+}\right] \triangleq\left[b_{1}^{-}, b_{1}^{+}\right] \times \cdots \times\left[b_{n}^{-}, b_{n}^{+}\right] \subset \mathbb{R}^{n},
$$

a small box around $\mathbf{0}$ :

$[\boldsymbol{\varepsilon}] \triangleq \prod_{k=1}^{m}\left[\varepsilon_{k}^{-}, \varepsilon_{k}^{+}\right] \subset \mathbb{R}^{m}$ with $-1 \ll \varepsilon_{k}^{-}<0<\varepsilon_{k}^{+} \ll 1$,

and a map $g:[\mathbf{b}] \rightarrow \mathbb{R}^{m}$, our objective is to sample the conditional vector $[\boldsymbol{X} \mid g(\boldsymbol{X}) \in[\boldsymbol{\varepsilon}]] .^{1}$ When the space dimension $n$ and the constraint dimension $p$ increase, the event $[g(\boldsymbol{X}) \in[\boldsymbol{\varepsilon}]]$ has very low probability. As a consequence, we are dealing here with a particular case of rare event simulation. Moreover, as $[g(\boldsymbol{X}) \in[\boldsymbol{\varepsilon}]]$ approximates a subvariety, it is foreseeable that the conditional vector $[\boldsymbol{X} \mid g(\boldsymbol{X}) \in[\boldsymbol{\varepsilon}]]$ is essentially and "continuously" multimodal.

In the survey (Morio et al., 2014), Morio, Balesdent et al. have evaluated the advantages and drawbacks of various rare event sampling methods. At this point, a comment may be done. In the litteracy, rare events are generally modelled by a function of risk being above an acceptable threshold: a rare event is of the form

\footnotetext{
a (iD) https://orcid.org/0000-0002-1460-4126

${ }^{1}$ In this paper, $[\boldsymbol{X} \mid g(\boldsymbol{X}) \in[\boldsymbol{\varepsilon}]]$ is used as an abbreviation for $[\boldsymbol{X} \mid \boldsymbol{X} \in[\mathbf{b}] \& g(\boldsymbol{X}) \in[\boldsymbol{\varepsilon}]]$, knowing that $g:[\mathbf{b}] \rightarrow \mathbb{R}^{m}$.
}

$[h(\boldsymbol{X})>\gamma]$, where $h$ maps to $\mathbb{R}$ and $P(h(\boldsymbol{X})>\gamma)$ is a very small probability to be evaluated. This formalism is quite general, and it is easy to rewrite event $[g(\boldsymbol{X}) \in[\boldsymbol{\varepsilon}]]$ under this form. However... The formalism $[h(\boldsymbol{X})>\boldsymbol{\gamma}]$ suggests that the simulation of a rare event is tightly related the the maximization of a function (function $h$ ). In practice, the maximizing set of a function is unimodal or somewhat multimodal. It is uncommon that this maximizing set is a subvariety.

Not surprizingly, many methods evaluated in (Morio et al., 2014) are working well when the rare event is unimodal or moderately multimodal. In the case of the cross-entropy method (Rubinstein and Kroese, 2004) for example, the multimodality has to be managed by the sampling law family. It is then possible, for example by a combination of EM algorithms and cross entropy minimization, to sample a moderately multimodal rare event (Dambreville, 2015). In the case of conditional vector $[\boldsymbol{X} \mid g(\boldsymbol{X}) \in[\boldsymbol{\varepsilon}]]$, these approaches do not work properly.

Good candidates for our sampling problem are some non parametric rare event simulation methods (Morio et al., 2014)(Cérou et al., 2012). Adaptive splitting techniques, as described in (Morio et al., 2014), are well suited for high dimensions and non linear systems. And although the method is designed for events of the form $[h[\boldsymbol{X}]>\gamma]$, it seems applicable to "subvariety" events of the form $[g(\boldsymbol{X}) \in[\boldsymbol{\varepsilon}]]$. However, as mentioned in (Morio et al., 2014), the approach needs an important simulation budget. In particular, the Markov transition kernel must be iterated several 
times during the resampling steps in order to get independent samples; the choice of the Markov transition kernel is crucial in design of the sampler. In our case, moreover, additional studies to take into account the subvariety structure can be benificial.

These arguments make the splitting technique promising for large-dimensional problems, but not necessarily for medium-dimensional problems. This article promotes an alternative approach based on a dichotomous exploration in order to sample conditionally to a subvariety characterized by a known function. By design, this approach produces independent samples and avoids the phenomenon of sample impoverishement. These properties make it particularly well suited for an accurate approximation of a law conditionally to the subvariety. However, the method must fight the curse of dimensionality. In practice, we will be faced with two orthogonal dimensional problems: the potential exponential increase in sampling exploration and the potential degeneracy of particle weights. This paper presents an approach and its perspectives, as well as a generic and weakly parameterized algorithm, allowing a good sampling performance for moderate dimensions (up to dimension 11 for now) and a balanced management of the dimensional issues.

Before completing this introduction, we should also mention a work, which addresses an issue which is connex to our concern. In (Bui Quang et al., 2016), Musso, Bui Quang and Le Gland proposed to combine Laplace method and particle filtering in order to dynamically estimate a state from partial measures with small observation noise. Actually, a partial measure with small observation noise implies a conditionning of the law by an approximated constraint. This is more or less the kind of problem we are dealing with. However, the approach proposed in (Bui Quang et al., 2016) is essentially unimodal, although it is possible to address some level of multimodality by a mixture of law (Musso et al., 2016). But for the moment this extension is far from meeting our requirements.

The paper is divided in four parts including the introduction. Section 2 introduces some concepts of interval analysis and derive some first ideas for a generic subvariety conditional sampler. Section 3 deepens the intuitions introduced in section 2 and presents an actually working generic algorithm. Few discussions about performance and perpectives follow. Section 4 presents tests and analyses. An example of application to black-box optimization is presented. Section 5 concludes.

\section{A NAIVE DICHOTOMOUS APPROACH FOR SAMPLING}

Interval analysis is a performing and accurate tool for dealing with constraints. Moreover, the approach provide a precise control on the approximation errors. Another interesting point is that intervals and boxes are relatively simple domains when dealing with probability distributions. For example, it is rather easy to evaluate the probability of a box being given the multivariate cumulative distribution function, especially for independent random variables. Boxes and intervals are also a good way to cope with poorly mastered random parameters. Thereby, Abdallah, Gning and Bonnifait proposed the box particle filter, mixing boxes into a particle filter, which resulted in better performance when dealing with non-white and biased measurement (Abdallah et al., 2008). Our work addresses a quite different issue. Essentially, boxes are not part of our data model, but we use the interval analysis as a tool and a guide for a dichotomous sampling on the subvariety.

\subsection{Some words about interval analysis}

It is not the purpose of this section to perform a good introduction on interval analysis (Alefeld and Mayer, 2000; Jaulin et al., 2001), and neither do we introduce properly the notion of paver for set inversion. Nevertheless, we refer to some key concepts of interval analysis, which are inspiring ideas for this paper. On the other hand, we absolutely do not introduce the technics of contractors (Chabert and Jaulin, 2009), but mention it as a possibility for improvement.

\subsubsection{Operators \& functions applied on intervals}

First at all, let us introduce some notations:

- $\mathbb{R}$ is the set of reals and $x, y, z$ are real variables.

- $[x] \triangleq\left[x^{-}, x^{+}\right],[y],[z]$ are notations for intervals. Bold notations $[\mathbf{x}] \triangleq \prod_{k=1}^{n}\left[x_{k}\right]=\prod_{k=1}^{n}\left[x_{k}^{-}, x_{k}^{+}\right]$and $\left[\boldsymbol{x}\left[\triangleq \prod_{k=1}^{n}\left[x_{k}^{-}, x_{k}^{+}[\right.\right.\right.$are used for boxes and halfopen boxes respectively.

- $[\mathbb{R}] \triangleq\left\{\left[x^{-}, x^{+}\right]:\left[x^{-}, x^{+}\right] \subset \mathbb{R}\right\}$ is the set of interval subsets of $\mathbb{R}$. $\left[\mathbb{R}^{n}\right] \triangleq\left\{\prod_{k=1}^{n}\left[x_{k}\right]: \forall k,\left[x_{k}\right] \in[\mathbb{R}]\right\}$ is the set of box subsets of $\mathbb{R}^{n}$.

- $\rho([x])=x^{+}-x^{-}$is the length of $[x] \in[\mathbb{R}]$. $\rho([\mathbf{x}])=\max _{1 \leq k \leq n} \rho\left(\left[x_{k}\right]\right)$ is the length of $[\mathbf{x}] \in\left[\mathbb{R}^{n}\right]$.

- $g: \mathbb{R} \rightarrow \mathbb{R}, g_{j}: \mathbb{R}^{k} \rightarrow \mathbb{R}, h: \mathbb{R}^{k} \rightarrow \mathbb{R}^{j}$ are notations for (multivariate) real functions, 
- $[g]:[\mathbb{R}] \rightarrow[\mathbb{R}],\left[g_{j}\right]:\left[\mathbb{R}^{k}\right] \rightarrow[\mathbb{R}],[h]:\left[\mathbb{R}^{k}\right] \rightarrow\left[\mathbb{R}^{j}\right]$ are notations for interval functions,

At this point, one should not confuse $\mathbf{x}$ and $[\mathbf{x}]$, nor should be confused $g$ and $[g]$, nor should be confused the notations $[g]([\mathbf{x}])$ and $g([\mathbf{x}])$ :

- $\mathbf{x} \in[\mathbf{x}]$ is equivalent to $\mathbf{x} \in \prod_{k=1}^{n}\left[x_{k}^{-}, x_{k}^{+}\right]$.

- $g([\mathbf{x}])=\{g(\mathbf{x}): \mathbf{x} \in[\mathbf{x}]\}$ is not the same as $[g]([\mathbf{x}])$ and is not even necessarily a box.

Nevertheless, we assume in this paper, that $g$ and $[g]$ are related by the following properties:

- $g([\mathbf{x}]) \subset[g]([\mathbf{x}])$ for any $[\mathbf{x}] \in\left[\mathbb{R}^{n}\right]$. In particular, $[g]$ is minimal when $[g]([\mathbf{x}])$ is the minimal box supset of $g([\mathbf{x}])$ for all $[\mathbf{x}] \in\left[\mathbb{R}^{n}\right]$.

- $[g]([\mathbf{x}]) \subset[g]([\mathbf{y}])$ when $[\mathbf{x}] \subset[\mathbf{y}]$.

- If $\rho([\mathbf{x}])$ vanishes, then $\rho([g]([\mathbf{x}]))$ vanishes:

$$
\rho(g([\mathbf{x}])) \underset{\rho([\mathbf{x}]) \rightarrow 0}{\longrightarrow} 0
$$

These properties expresses that $[g]$ implies a bound on the error propagated by $g$, and this bound has good convergence behavior in regards to the error.

Before going further, let us consider how to build the interval functions on some common examples:

1. Reference functions, $g \in\left\{\ln , \exp , \sin , \cos , .^{n}, \ldots\right\}$, are continuous onto $\mathbb{R}$, so that $g([x]) \in[\mathbb{R}]$ for all $[x] \in[\mathbb{R}]$. Then, it is optimal to set $[g]([x])=g([x])$ for all $[x] \in[\mathbb{R}]$. As a consequence, the interval functions are easily optimaly implementable for most reference functions. Here are some incomplete examples of definitions:

$[\ln ]([x]) \triangleq\left[\ln \left(x^{-}\right), \ln \left(x^{+}\right)\right],[1 /][x] \triangleq \frac{1}{[x]}=\left[\frac{1}{x^{+}}, \frac{1}{x^{-}}\right]$,

$\left.[x]]^{n}\right] \triangleq[x]^{n}=\left\{\begin{array}{l}{\left[x_{-}^{n}, x_{+}^{n}\right] \text { for } n>0,} \\ {\left[x_{+}^{n}, x_{-}^{n}\right] \text { for } n<0,}\end{array}\right.$

$[\cos ][x] \triangleq\left\{\begin{array}{l}{\left[\cos \left(x^{-}\right), \cos \left(x^{+}\right)\right] \text {for }[x] \subset[-\pi, 0]} \\ {\left[\cos \left(x^{+}\right), \cos \left(x^{-}\right)\right] \text {for }[x] \subset[0, \pi]} \\ {\left[\min \left(\cos \left(x^{-}\right), \cos \left(x^{+}\right)\right), 1\right]} \\ \quad \text { for } 0 \in[x] \subset[-\pi, \pi] \\ \text { etc. }\end{array}\right.$

2. Minimal interval functions for classical operators $+, \cdot,-, /$ are also easily defined. For example:

$$
[x][+][y] \triangleq[x]+[y]=\left[x^{-}+y^{-}, x^{+}+y^{+}\right]
$$

3. Function $g$ defined by $g(\theta)=(\cos (\theta), \sin (\theta))$, is an example where $g([\theta]) \notin\left[\mathbb{R}^{2}\right]$. One would rather define $[g]([\theta])=[\cos ]([\theta]) \times[\sin ]([\theta])$ which is a strict supset of $g([\theta])$ in general. By the way, this construction is an illustration on how the interval functions of reference are used to define complex interval functions straightforwardly.
There is no unicity in the construction of $[g]$. Let us consider the case of function $g: \theta \mapsto \cos ^{2} \theta+\sin ^{2} \theta$. Then, there are two obvious definitions for $[g]$.

1. By using the reference functions $\cos , \sin , .^{2}$ and + one may derive:

$$
[g]([\theta])=([\cos ]([\boldsymbol{\theta}]))^{2}+([\sin ]([\boldsymbol{\theta}]))^{2} .
$$

For example, we compute: $[g]\left(\left[0, \frac{\pi}{2}\right]\right)=[0,2]$ which is a bad error bound on theoretical value 1 . Now, we also compute: $[g]\left(\left[-\frac{1}{10}, \frac{1}{10}\right]\right) \simeq$ $[0.99,1.01]$ which is a tight error bound on 1 . This example hold confirmation that $[g]([\theta])$ has a good behavior for small boxes $[\theta]$.

2. By noticing that $\cos ^{2} \theta+\sin ^{2} \theta=1$, it is optimal to define $[g]([\theta])=[1,1]$.

Although approach 2 gives the best solution, in practice approach 1 is prefered since it is generic, it is based on already implemented functions of reference, and it provides a way to construct automatically $[g]$ without any specific knowledge.

\subsubsection{Subpaving}

As $[g]$ implies a bound on the error propagated by $f$ with good convergence behavior, it may be used combined with a dichotomous process to produce a subpaving which efficiently approximate a set inversion $g^{-1}([\mathbf{y}])$. The example of figure 1 is kindly given by professor Jaulin. It shows a resulting subpaving which approximates a set inversion. The dichotomous

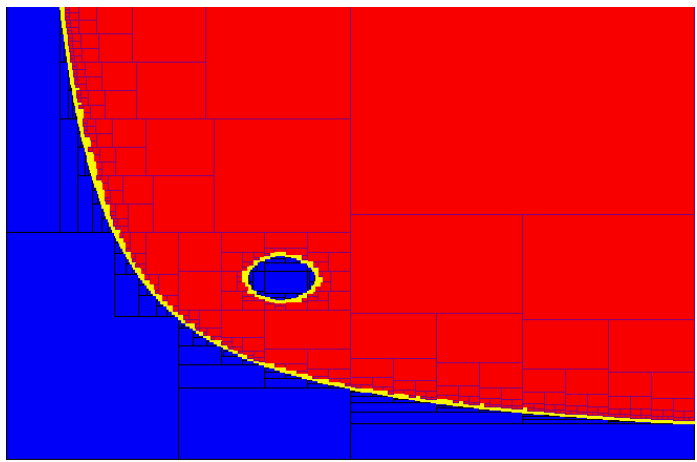

Figure 1: Example of set inversion

nature of the decomposition is clear. A bisection process is iterated starting from the main box; at each iteration, sub-boxes $[\mathbf{x}]$ are tested against the constraint $g([\mathbf{x}]) \subset[\mathbf{y}]$. Then three cases arise:

case a: $g([\mathbf{x}]) \subset[\mathbf{y}]$, then $[\mathbf{x}]$ is among red boxes, which constitute a subpaving of $g^{-1}([\mathbf{y}])$.

case b: $g([\mathbf{x}]) \cap[\mathbf{y}]=\emptyset$, then $[\mathbf{x}]$ is among blue boxes, which constitute a subpaving of $\mathbb{R}^{n} \backslash g^{-1}([\mathbf{y}])$.

case c: Otherwise, bisection has to be repeated on $[\mathbf{x}]$ until sufficient convergence (yellow color). 
Property (1) plays a key role in the decomposition process, ensuring that case $\mathbf{a}$ or case $\mathbf{b}$ are finally achieved when sub-boxes $[\mathbf{x}]$ are sufficiently small and sufficiently far from the frontier of set $g^{-1}([\mathbf{y}])$.

\subsection{Toward a generic sampling method based on a dichotomous approach}

Now, we settle the hypotheses mentioned in introduction. Let $\mu$ be Borel measure on $\mathbb{R}^{n}$. It is given:

- a random vector $\boldsymbol{X}$ defined on $\mathbb{R}^{n}$ characterized by a bounded density $f_{\boldsymbol{X}}$. The cumulative distribution function $F_{\boldsymbol{X}}$ of $\boldsymbol{X}$ defined for all $\mathbf{x} \in \mathbb{R}^{n}$ by:

$$
F_{\boldsymbol{X}}(\mathbf{x})=P(\boldsymbol{X} \leq \mathbf{x})=\int_{\mathbf{y} \leq \mathbf{x}} f_{\boldsymbol{X}}(\mathbf{y}) \mu(\mathbf{d y})
$$

is assumed to be easily computable,

- a box $[\mathbf{b}] \in\left[\mathbb{R}^{n}\right]$,

- a small box $[\boldsymbol{\varepsilon}] \in\left[\mathbb{R}^{m}\right]$,

- a continuous map $g:[\mathbf{b}] \rightarrow \mathbb{R}^{p}$ defined by composing functions and operators of reference,

- $[g]$ derived from $g$ by composing the related interval functions and operators of reference.

\subsubsection{Cumulative functions and boxes}

We point out that it is easy to compute $P(\boldsymbol{X} \in[\boldsymbol{y}])$ or to sample $[\boldsymbol{X} \mid \boldsymbol{X} \in[\boldsymbol{y}]]$ when $F_{\boldsymbol{X}}$ is available. Thus, these features are taken for granted in this paper.

First, it is well known that $P(\boldsymbol{X} \in[\boldsymbol{y}])$ is litteraly computable from $F_{\boldsymbol{X}}$ :

$P(\boldsymbol{X} \in[\boldsymbol{y}])=\sum_{\boldsymbol{\sigma} \in\{-1,+1\}^{n}} F_{\boldsymbol{X}}\left(\left(y_{k}^{\operatorname{sgn}\left(\sigma_{k}\right)}\right)_{1 \leq k \leq n}\right) \prod_{k=1}^{n} \sigma_{k}$,

where $\operatorname{sgn}(1) \triangleq+$ and $\operatorname{sgn}(-1) \triangleq-$. When the components of $\boldsymbol{X}$ are jointly independent, the computation is dramatically accelerated by factoring $F_{\boldsymbol{X}}$ and computing time becomes linear with the dimension.

Sampling $[\boldsymbol{X} \mid \boldsymbol{X} \in[\boldsymbol{y}]]$ is easily done by means of $F_{\boldsymbol{X}}$ :

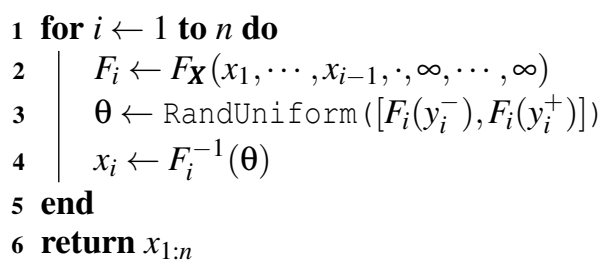

The principle is to iteratively apply the inverse transform sampling method to $F_{i}$, the marginal in $x_{i}$ of the cumulative function conditionally to the already sampled components $x_{1}, \cdots, x_{i-1}$.

\subsubsection{Subpaving based sampling}

Now assume that set $g^{-1}([\boldsymbol{\varepsilon}])$ has been approximated (by default) by a subpaving as shown in figure 1. In other words, there is $\mathbb{P} \subset\left[R^{n}\right]$ such that:

- For all $[\boldsymbol{x}],[\boldsymbol{y}] \in\left[R^{n}\right]$, boxes $[\boldsymbol{x}]$ and $[\boldsymbol{y}]$ are disjoint except possibly on the edges,

- $g^{-1}([\boldsymbol{\varepsilon}]) \simeq \sqcup_{\mathbb{P}} \subseteq g^{-1}([\boldsymbol{\varepsilon}])$, where $\sqcup_{\mathbb{P}} \triangleq \bigsqcup_{[\boldsymbol{x}] \in \mathbb{P}}[\boldsymbol{x}[$.

Considering Borel measure $\mu$ on $\mathbb{R}^{n}$, the quality of the approximation may be quantified by set measures:

$$
\alpha_{\mathbb{P}}=\mu\left(g^{-1}([\boldsymbol{\varepsilon}]) \backslash \sqcup_{\mathbb{P}}\right)=\mu\left(g^{-1}([\boldsymbol{\varepsilon}])\right)-\sum_{[\boldsymbol{x}] \in \mathbb{P}} \mu([\boldsymbol{x}[) .
$$

Smaller is $\alpha_{\mathbb{P}}$, better is the approximation. Interval based set inversion algorithms are able to reach an arbitrary precision, at least for small dimensions ( 2 or 3 are reasonable).

Now, for all $\boldsymbol{y} \in \mathbb{R}^{n}$, it happens that:

$$
\begin{aligned}
f_{\boldsymbol{X} \mid \boldsymbol{X} \in \sqcup_{\mathbb{P}}}(\boldsymbol{y})=\frac{f_{\boldsymbol{X}}(\boldsymbol{y}) \boldsymbol{\delta}_{\boldsymbol{y} \in \sqcup_{\mathbb{P}}}}{P\left(\boldsymbol{X} \in \sqcup_{\mathbb{P}}\right)} \\
=\frac{f_{\boldsymbol{X}}(\boldsymbol{y}) \sum_{[\boldsymbol{x}] \in \mathbb{P}} \delta_{\boldsymbol{y} \in[\boldsymbol{x}[} \sum_{[\boldsymbol{x}] \in \mathbb{P}} P(X \in[\boldsymbol{x}[)}{P}=\frac{\sum_{[\boldsymbol{x}] \in \mathbb{P}} P\left(X \in \left[\boldsymbol{x}[) f_{\boldsymbol{X} \mid \boldsymbol{X} \in[\boldsymbol{x}[}(\boldsymbol{y})\right.\right.}{\sum_{[\boldsymbol{x}] \in \mathbb{P}} P(X \in[\boldsymbol{x}[)} \\
=\sum_{[\boldsymbol{x}] \in \mathbb{P}} \frac{P(X \in[\boldsymbol{x}])}{\sum_{[\boldsymbol{x}] \in \mathbb{P}} P(X \in[\boldsymbol{x}])} f_{\boldsymbol{X} \mid \boldsymbol{X} \in[\boldsymbol{x}[(\mathbf{y})}
\end{aligned}
$$

where $\delta_{\text {true }}=1$ and $\delta_{\text {false }}=0$ else.

Since $f_{\boldsymbol{X}}$ is bounded, it is easily derived from (2):

$$
f_{\boldsymbol{X} \mid \boldsymbol{X} \in \sqcup_{\mathbb{P}}}(\boldsymbol{y}) \underset{\alpha_{\mathbb{P}} \rightarrow 0}{\longrightarrow} f_{\boldsymbol{X} \mid \boldsymbol{X} \in g^{-1}([\boldsymbol{\varepsilon}]}(\boldsymbol{y})
$$

On the other hand, equation (3) shows clearly that $f_{\boldsymbol{X} \mid \boldsymbol{X} \in \sqcup_{\mathbb{P}}}$ may be sampled by applying two steps: first sample a box $[\boldsymbol{x}] \in \mathbb{P}$ according to the discrete probability $\frac{P(X \in[\boldsymbol{x}])}{\sum_{[\boldsymbol{x}] \in \mathbb{P}} P(X \in[\boldsymbol{x}])}$, then sample $\boldsymbol{y}$ by the conditional

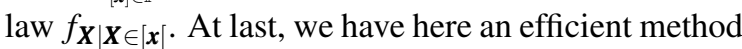
for sampling $[\boldsymbol{X} \mid g(\boldsymbol{X}) \in[\boldsymbol{\varepsilon}]]$ (algorithm 1):

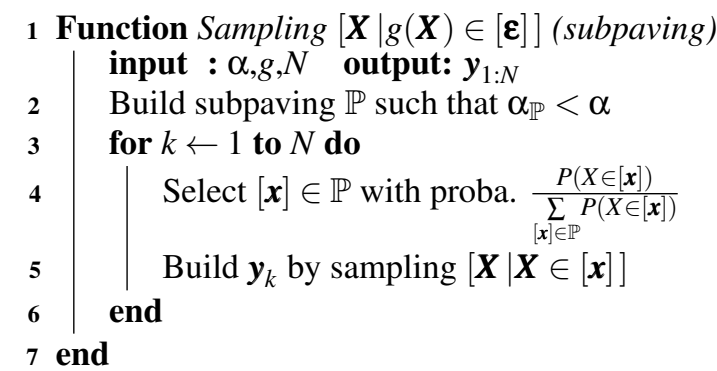

Algorithm 1: Based on a subpaving

The approach is efficient on rare events of the form 
$[\boldsymbol{X} \mid g(\boldsymbol{X}) \in[\boldsymbol{\varepsilon}]]$. However, this immediate application of the interval-based inversion is only applicable to rather small dimensions, since the size of the subpaving should increase exponentially. Taking inspiration of this preliminary approach, we address now the sampling problem in higher dimensions.

\subsubsection{Naive dichotomous sampling}

A key point of algorithm 1 is to be able to sample a box $[\boldsymbol{x}]$ of a subpaving of $g^{-1}([\boldsymbol{\varepsilon}])$. It is noticeable that we do not need to build the full subpaving; if we were able to construct a box $[\boldsymbol{x}]$ of the subpaving on demand, together with its relative weight within the subpaving, then we would be able to build sample $\boldsymbol{y}$.

To begin with, we define the notion of cut:

- A cut of box $[\boldsymbol{x}] \in\left[\mathbb{R}^{n}\right]$ is a pair $([\boldsymbol{l}],[\boldsymbol{r}]) \in\left[\mathbb{R}^{n}\right]^{2}$ such that $[\boldsymbol{l}[\cap[\boldsymbol{r}[=\emptyset$ and $[\boldsymbol{l}[\sqcup[\boldsymbol{r}[=[\boldsymbol{x}[$.

- A bisection is a cut $([\boldsymbol{l}],[\boldsymbol{r}])$ such that $[\boldsymbol{l}]$ and $[\boldsymbol{r}]$ are same-sized.

In general, bisections are often used in dichotomous processes, as there is a garanty of exponential volume decrease of the search area. ${ }^{2}$ Here, we speak in terms of cuts, which are more general, being implied that an appropriate management of the box length is made in order to ensure the convergence.

We assume here that a weighting function is available:

$$
\omega_{[\boldsymbol{x}]} \simeq P(\boldsymbol{X} \in[\boldsymbol{x}] \& g(\boldsymbol{X}) \in[\boldsymbol{\varepsilon}]) .
$$

Algorithm 2 implicitly builds a partial subpaving, and actually produces a weighted particle cloud as a result of the sampling of $[\boldsymbol{X} \mid g(\boldsymbol{X}) \in[\boldsymbol{\varepsilon}]]$ :

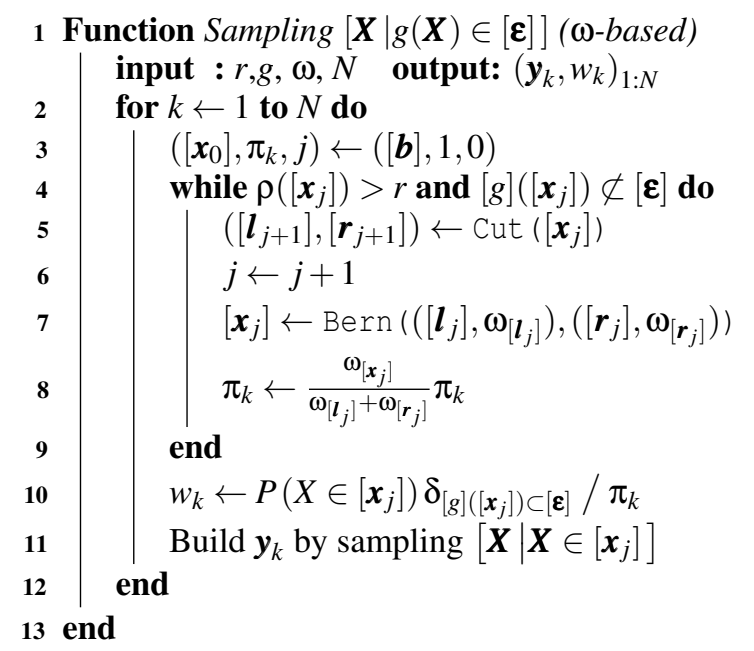

Algorithm 2: Based on a weighting function

\footnotetext{
${ }^{2}$ Though, bisections do not guarantee a decrease in length $\rho$ unless the cuts are managed in all directions.
}

The algorithm iterates in a for loop the same sampling process. It iterates the following successive steps until $\left[\boldsymbol{x}_{j}\right]$ is sufficiently small (ie. $\rho\left(\left[\boldsymbol{x}_{j}\right]\right) \leq r$ ) or is inside an implied suppaving $\left(i e .[g]\left(\left[\boldsymbol{x}_{j}\right]\right) \subset[\boldsymbol{\varepsilon}]\right):^{3}$

- Cut $\left[\boldsymbol{x}_{j}\right]$ by means of Cut $\left(\left[\boldsymbol{x}_{j}\right]\right)$. This function is designed so as to ensure that $\rho\left(\left[\boldsymbol{x}_{j}\right]\right)$ vanishes,

- Select randomly one of the cut $\left(\left[\boldsymbol{l}_{j}\right],\left[\boldsymbol{r}_{j}\right]\right)$ in proportion to their weight, by mean of Bernoulli process Bern $\left(\left(\left[\boldsymbol{l}_{j}\right], \omega_{\left[\boldsymbol{l}_{j}\right]}\right),\left(\left[\boldsymbol{r}_{j}\right], \omega_{\left[\boldsymbol{r}_{j}\right]}\right)\right)$,

- Update $\pi_{k}$ which computes the processed probability of $\left[\boldsymbol{x}_{j}\right]$ in regards to the Bernoulli sequence.

Assume that $J$ is the last value reached by parameter $j$ after the while loop. Then, the corrected weight $w_{k}=\frac{1}{\pi_{k}} P\left(X \in\left[\boldsymbol{x}_{J}\right]\right) \delta_{[g]\left(\left[\boldsymbol{x}_{J}\right]\right) \subset[\boldsymbol{\varepsilon}]}$ is computed for $\left[\boldsymbol{x}_{J}\right]$ and for $\boldsymbol{y}_{k}$, and $\boldsymbol{y}_{k}$ is sampled from $\left[\boldsymbol{x}_{J}\right]$.

Notice that $w_{k}$ is zeroed when $[g]\left(\left[\boldsymbol{x}_{J}\right]\right) \not \subset[\boldsymbol{\varepsilon}]$, which implies that only the boxes $\left[\boldsymbol{x}_{J}\right]$ of a subpaving of $g^{-1}([\boldsymbol{\varepsilon}])$ are actually considered. Combined with loop constraint $\rho\left(\left[\boldsymbol{x}_{J}\right]\right)>r$ and $[g]\left(\left[\boldsymbol{x}_{j}\right]\right) \not \subset[\boldsymbol{\varepsilon}]$, it follows that a subpaving of $g^{-1}([\boldsymbol{\varepsilon}])$ is implicitely and partially built during the sampling process.

When $[g]\left(\left[\boldsymbol{x}_{J}\right]\right) \subset[\boldsymbol{\varepsilon}]$, we have $w_{k}=\frac{P\left(X \in\left[\boldsymbol{x}_{J}\right]\right)}{\pi_{k}}$ where $\pi_{k}$ evaluates the processed probability for $\left[\boldsymbol{x}_{J}\right]$. As a result, the weighted particles $\left(\boldsymbol{y}_{k}, w_{k}\right)$ provide an unbiased estimation of $f_{\boldsymbol{X} \mid g(\boldsymbol{X}) \in[\boldsymbol{\varepsilon}]}$ in a subpaving of $g^{-1}([\boldsymbol{\varepsilon}])$. It is not the same at the border of $g^{-1}([\boldsymbol{\varepsilon}])$, but this case is neglected. However, the sampler is not at all efficient when considering its variance.

First, let us consider a case which works perfectly. Assume $\omega_{[\boldsymbol{x}]}=P(\boldsymbol{X} \in[\boldsymbol{x}] \& g(\boldsymbol{X}) \in[\boldsymbol{\varepsilon}])$. In this ideal case, the weight along a while loop is computed by:

$$
\begin{aligned}
\frac{\omega_{\left[\boldsymbol{x}_{j}\right]}}{\omega_{\left[\boldsymbol{l}_{j}\right]}+\omega_{\left[\boldsymbol{r}_{j}\right]}} & =\frac{P\left(\boldsymbol{X} \in\left[\boldsymbol{x}_{j}\right] \& g(\boldsymbol{X}) \in[\boldsymbol{\varepsilon}]\right)}{P\left(\boldsymbol{X} \in\left[\boldsymbol{l}_{j}\right] \cup\left[\boldsymbol{r}_{j}\right] \& g(\boldsymbol{X}) \in[\boldsymbol{\varepsilon}]\right)} \\
& =\frac{P\left(\boldsymbol{X} \in\left[\boldsymbol{x}_{j}\right] \& g(\boldsymbol{X}) \in[\boldsymbol{\varepsilon}]\right)}{P\left(\boldsymbol{X} \in\left[\boldsymbol{x}_{j-1}\right] \& g(\boldsymbol{X}) \in[\boldsymbol{\varepsilon}]\right)},
\end{aligned}
$$

and then:

$$
\pi_{k}=\prod_{j=1}^{J} \frac{\omega_{\left[\boldsymbol{x}_{j}\right]}}{\omega_{\left[l_{j}\right]}+\omega_{\left[\boldsymbol{r}_{j}\right]}}=\frac{P\left(\boldsymbol{X} \in\left[\boldsymbol{x}_{J}\right] \& g(\boldsymbol{X}) \in[\boldsymbol{\varepsilon}]\right)}{P(\boldsymbol{X} \in[\boldsymbol{b}] \& g(\boldsymbol{X}) \in[\boldsymbol{\varepsilon}])} .
$$

Three cases potentially arise:

- $[g]\left(\left[\boldsymbol{x}_{J}\right]\right) \subset[\boldsymbol{\varepsilon}]$, ie. $\left[\boldsymbol{x}_{J}\right]$ is in an implied subpaving. Now $P\left(\boldsymbol{X} \in\left[\boldsymbol{x}_{J}\right] \& g(\boldsymbol{X}) \in[\boldsymbol{\varepsilon}]\right)=P\left(\boldsymbol{X} \in\left[\boldsymbol{x}_{J}\right]\right)$, as a consequence of $g\left(\left[\boldsymbol{x}_{J}\right]\right) \subset[g]\left(\left[\boldsymbol{x}_{J}\right]\right) \subset[\boldsymbol{\varepsilon}]$. Then:

$$
\begin{aligned}
w_{k} & =\frac{P\left(X \in\left[\boldsymbol{x}_{J}\right]\right) \delta_{[g]\left(\left[\boldsymbol{x}_{J}\right]\right) \subset[\boldsymbol{\varepsilon}]}}{\frac{P\left(\boldsymbol{X} \in\left[\boldsymbol{x}_{J}\right] \& g(\boldsymbol{X}) \in[\boldsymbol{\varepsilon}]\right)}{P(\boldsymbol{X} \in[\boldsymbol{b}] \& g(\boldsymbol{X}) \in[\boldsymbol{\varepsilon}])}}=\frac{P\left(X \in\left[\boldsymbol{x}_{J}\right]\right)}{\frac{P\left(\boldsymbol{X} \in\left[\boldsymbol{x}_{J}\right]\right)}{P(\boldsymbol{X} \in[\boldsymbol{b}] \& g(\boldsymbol{X}) \in[\boldsymbol{\varepsilon}])}} \\
& =P(\boldsymbol{X} \in[\boldsymbol{b}] \& g(\boldsymbol{X}) \in[\boldsymbol{\varepsilon}]) \triangleq P(g(\boldsymbol{X}) \in[\boldsymbol{\varepsilon}])
\end{aligned}
$$

\footnotetext{
${ }^{3}$ Recall that $[g]\left(\left[\boldsymbol{x}_{j}\right]\right)$ is computable while $g\left(\left[\boldsymbol{x}_{j}\right]\right)$ is not.
} 
- $[g]\left(\left[\boldsymbol{x}_{J}\right]\right) \cap[\boldsymbol{\varepsilon}] \neq \emptyset$ but $[g]\left(\left[\boldsymbol{x}_{J}\right]\right) \not \subset[\boldsymbol{\varepsilon}]$, ie. $\left[\boldsymbol{x}_{J}\right]$ is within the border of the implied subpaving. Then $w_{k}=0$. Since we are at the border, these lost cases are negligible for small precision $r$.

- $[g]\left(\left[\boldsymbol{x}_{J}\right]\right) \cap[\boldsymbol{\varepsilon}]=\emptyset$, ie. $\left[\boldsymbol{x}_{J}\right]$ is ouside the implied subpaving and its border. Then $g\left(\left[\boldsymbol{x}_{J}\right]\right) \cap[\boldsymbol{\varepsilon}]=\emptyset$ and $\omega_{\left[x_{J}\right]}=P\left(\boldsymbol{X} \in\left[\boldsymbol{x}_{J}\right] \& g(\boldsymbol{X}) \in[\boldsymbol{\varepsilon}]\right)=0$. Case is simply impossible from the Bernoulli process.

Equation (5) shows that the sampling process results in a cloud of same-weight particles over the implied subpaving. Other cases (rejections) are negligeable. Here we have a sampler of $[\boldsymbol{X} \mid g(\boldsymbol{X}) \in[\boldsymbol{\varepsilon}]]$ with the best variance performance in regards to the number of particles. But this is achieved only when $\omega_{[\boldsymbol{x}]}=P(\boldsymbol{X} \in[\boldsymbol{x}] \& g(\boldsymbol{X}) \in[\boldsymbol{\varepsilon}])$, and of course such exact weighting function is almost never available.

Why it does not work in general cases? In the cases where $\omega_{[\boldsymbol{x}]} \neq P(\boldsymbol{X} \in[\boldsymbol{x}] \& g(\boldsymbol{X}) \in[\boldsymbol{\varepsilon}])$, the accumulated error will explode with the dimension, which will result in dramatically uneven weights on the particles. The resulting weighted particles cloud is then useless for practical applications.

\section{A DICHOTOMOUS APPROACH FOR SAMPLING}

Algorithms 1 and 2 illustrate the two main dimensional issues, that we have to deal with. And these approaches are complementary:

- By building a complete subpaving of $g^{-1}([\boldsymbol{\varepsilon}])$, algorithm 1 makes possible a direct sampling of $[\boldsymbol{X} \mid g(\boldsymbol{X}) \in[\boldsymbol{\varepsilon}]]$, and incidently an accurate computation of $P(\boldsymbol{X} \in[\boldsymbol{x}] \& g(\boldsymbol{X}) \in[\boldsymbol{\varepsilon}])$. However, this construction of a complete subpaving is only possible for small dimensions.

- Algorithm 2 avoids the construction of a complete subpaving. Instead, it builds the boxes of an implied subpaving on demand throughout the sampling iteration. However, the algorithm is inefficient unless the weighting function $\omega_{[x]}$ is a good approximation of $P(\boldsymbol{X} \in[\boldsymbol{x}] \& g(\boldsymbol{X}) \in[\boldsymbol{\varepsilon}])$. This condition is not accessible in general.

We propose now an intermediate approach which:

- keeps history of the subpaving construction throughout the sampling process,

- use this history to build an improved estimate of $P(\boldsymbol{X} \in[\boldsymbol{x}] \& g(\boldsymbol{X}) \in[\boldsymbol{\varepsilon}])$.

By these tricks, it is expected that the sampling precision will increase with the number of samples. In order to avoid useless exploration, we also truncate the dichotomous process on the basis of some predictive assessment of final weight $y_{k}$. Thus, the algorithm tends to favor breadth search instead of depth search at the early stages of the sampling process.

\subsection{Implementing some containment of the curse of dimension}

From now on, it is assumed that:

$$
0 \leq \omega_{[x]} \leq P(X \in[x]),
$$

and that:

$$
\omega_{[x]}=\left\{\begin{array}{l}
0 \text { if }[g]([\boldsymbol{x}]) \cap[\boldsymbol{\varepsilon}]=\emptyset, \\
P(X \in[\boldsymbol{x}]) \text { if }[g]([\boldsymbol{x}]) \subset[\boldsymbol{\varepsilon}] .
\end{array}\right.
$$

Algorithm 3 is an evolution of algorithm 2. In addition, it builds an history of the cuts, stored in map cuts, and computes dynamically from this history an improved weighting function, stored in map omg :

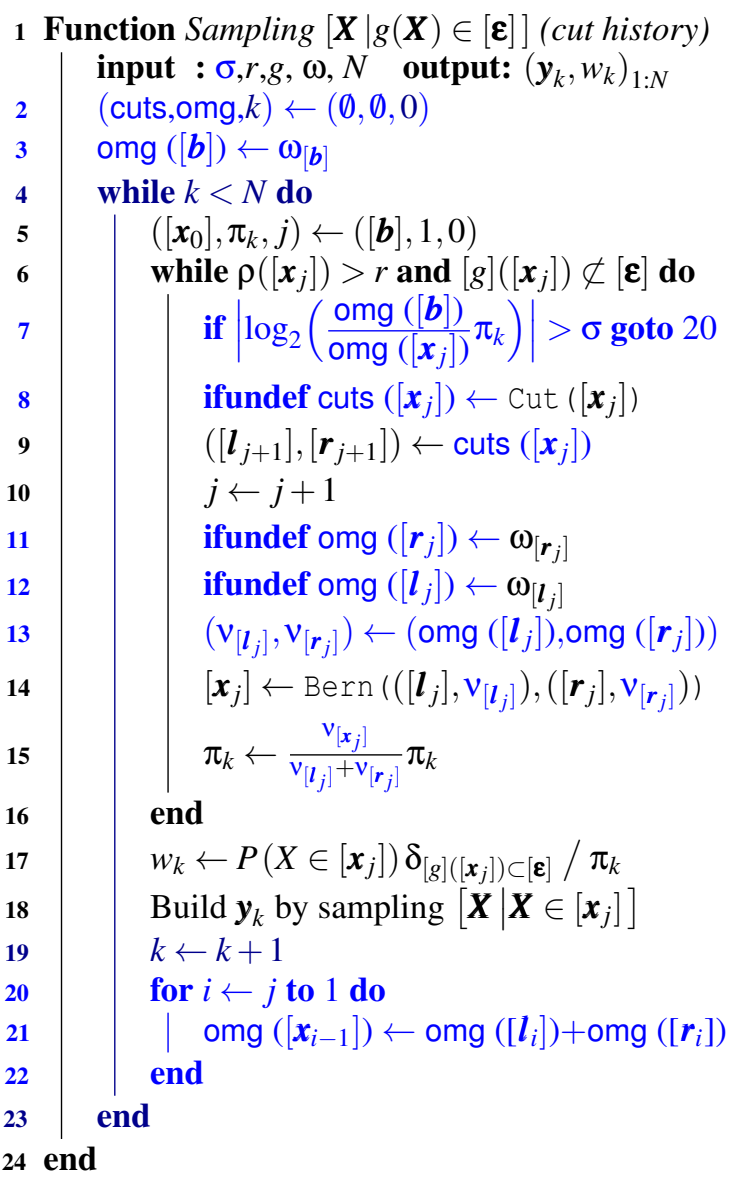

Algorithm 3: Based on cuts history

The lines of this algorithm are colored in black, in blue or in dark blue. Black lines are directly inherited from algorithm 2 . Blue lines are new additions to the 
previous algorithm. Dark blue lines $(4,19,23$ and 2 partially) correspond to the inside while loop:

$$
k \leftarrow 0 \text { while } k<N \text { do } \cdots k \leftarrow k+1 \cdots \text { end }
$$

Essentially, it is a detailed rewrite of for loop in algorithm 2, which allows a better control on the incrementation of variable $k$.

Variable cuts is a dictionary which is used to register the history of computed cuts. At start, cuts is defined empty (line 2). For a given box $\left[\boldsymbol{x}_{j}\right]$, the cut on $\left[\boldsymbol{x}_{j}\right]$ is computed only once, if it is computed, by line 8 :

$$
\text { ifundef cuts }\left(\left[\boldsymbol{x}_{j}\right]\right) \leftarrow \operatorname{Cut}\left(\left[\boldsymbol{x}_{j}\right]\right)
$$

Keyword ifundef tests if cuts $\left(\left[\boldsymbol{x}_{j}\right]\right)$ is defined. If it is still undefined, then cuts $\left(\left[\boldsymbol{x}_{j}\right]\right)$ is set to Cut $\left(\left[\boldsymbol{x}_{j}\right]\right)$.

Variable omg is a dictionary which records the weighting function and its possible updates, when needed. At start, omg is only defined for $[\boldsymbol{b}]$ and is set to $\omega_{[\boldsymbol{b}]}$ (lines 1 and 3 ). Variable omg $\left(\left[\boldsymbol{r}_{j}\right]\right)$ is set to $\omega_{\left[\boldsymbol{r}_{j}\right]}$, if it has not been initialized yet (line 11). The same is done for variable omg $\left(\left[\boldsymbol{l}_{j}\right]\right)$ at line 12 . When the cuts sequence is done (second while), then the weighting function is updated by the for loop (lines $20,21,22)$. This ensures that omg $([\boldsymbol{x}])$ is computed as the sum of the weights omg $([z])$ of the leaves $[z]$ of the cuts tree rooted on $[\boldsymbol{x}]$. Then, property (7) ensures that omg $([\boldsymbol{x}])$ gets closer to $P(\boldsymbol{X} \in[\boldsymbol{x}] \& g(\boldsymbol{X}) \in[\boldsymbol{\varepsilon}])$ when the cuts tree rooted on $[\boldsymbol{x}]$ gets more refined.

Algorithm 3 is similar to algorithm 2, except that:

- the cut $\left(\left[\boldsymbol{l}_{j+1}\right],\left[\boldsymbol{r}_{j+1}\right]\right)$ is recovered from the history, when it is possible (line 9 ),

- the cut choice is done by means of updated weights $\left(\boldsymbol{v}_{\left[\boldsymbol{l}_{j}\right]}, \mathrm{v}_{\left[\boldsymbol{r}_{j}\right]}\right) \triangleq\left(\right.$ omg $\left(\left[\boldsymbol{l}_{j}\right]\right)$,omg $\left.\left(\left[\boldsymbol{r}_{j}\right]\right)\right)$.

There is an interesting property here. Assume that $J$ is the last value reached by $j$ and that $J^{\prime}<J$ is such that omg $\left(\left[\boldsymbol{l}_{j}\right]\right)$ and omg $\left(\left[\boldsymbol{r}_{j}\right]\right)$ are already defined for all $1 \leq j \leq J^{\prime}$. The weighting functions are updated in these cases. Then, it comes for all $1 \leq j \leq J^{\prime}$ that:

$$
\operatorname{omg}\left(\left[\boldsymbol{x}_{j-1}\right]\right)=\operatorname{omg}\left(\left[\boldsymbol{l}_{j}\right]\right)+\operatorname{omg}\left(\left[\boldsymbol{r}_{j}\right]\right) .
$$

The computation of $\pi_{k}$ is then simplified:

$$
\begin{aligned}
\pi_{k} & =\prod_{j=1}^{J^{\prime}} \frac{v_{\left[\boldsymbol{x}_{j}\right]}}{v_{\left[\boldsymbol{l}_{j}\right]}+\mathrm{v}_{\left[\boldsymbol{r}_{j}\right]}} \prod_{j=J^{\prime}+1}^{J} \frac{\mathrm{v}_{\left[\boldsymbol{x}_{j}\right]}}{\mathrm{v}_{\left[\boldsymbol{l}_{j}\right]}+\mathrm{v}_{\left[\boldsymbol{r}_{j}\right]}} \\
& =\prod_{j=1}^{J^{\prime}} \frac{\mathrm{v}_{\left[\boldsymbol{x}_{j}\right]}}{\mathrm{v}_{\left[\boldsymbol{x}_{j-1}\right]}} \prod_{j=J^{\prime}+1}^{J} \frac{\mathrm{v}_{\left[\boldsymbol{x}_{j}\right]}}{\mathrm{v}_{\left[\boldsymbol{l}_{j}\right]}+\mathrm{v}_{\left[\boldsymbol{r}_{j}\right]}} \\
& =\frac{\mathrm{v}_{\left[\boldsymbol{x}_{J^{\prime}}\right]}}{\mathrm{v}_{[\boldsymbol{b}]}} \prod_{j=J^{\prime}+1}^{J} \frac{\mathrm{v}_{\left[\boldsymbol{x}_{j}\right]}}{\mathrm{v}_{\left[\boldsymbol{l}_{j}\right]}+\mathrm{v}_{\left[\boldsymbol{r}_{j}\right]}} .
\end{aligned}
$$

Thus, the error on $\pi_{k}$ grows exponentially only within the newly explored cuts, that is here from $J^{\prime}+1$ to $J$. This is a reason for setting a certain restriction on the depth-oriented aspect of this sampling process. Another good reason is to prevent degenerate particle weights, $w_{k}$, which are actually useless. Algorithm 3 thus implements some code (line 7) for testing the degeneracy of $\pi_{k}$ and eventually restarting the sampling loop (second while):

$$
\text { if }\left|\log _{2}\left(\frac{\text { omg }([\boldsymbol{b}])}{\operatorname{omg}\left(\left[\boldsymbol{x}_{j}\right]\right)} \pi_{k}\right)\right|>\sigma \text { goto } 20
$$

This code tests the logarithmic distance between the weight of $\left[\boldsymbol{x}_{j}\right]$, omg $\left(\left[\boldsymbol{x}_{j}\right]\right)$, and the weight resulting from the sampling process, omg $([\boldsymbol{b}]) \boldsymbol{\pi}_{k}$. If this distance is higher than $\sigma$, then the loop is stopped by going to line 20. By doing that, the incrementation of $k$ is skipped, so that the sampling loop is restarted for the same indice $k$. However, the update of variable omg is done, and of course, the history of cuts stays incremented. So, although the sampling loop has been interrupted in this case, the sampling structure has been upgraded. This results in an adaptive process which will balance depth and breadth explorations when running the sampling. Breadth exploration is favored on the first sampling iterations, but the tendency becomes inverted after several samples.

\subsection{Practical implementation}

Algorithm 3 draws the main principles of our sampling method. Of course, some details are not described there, although they are necessary for a practical implementation. Some points may be mentioned:

- We implement the following definition of $\omega$ :

$$
\omega_{[x]}=\frac{\mu([g]([\boldsymbol{x}]) \cap[\boldsymbol{\varepsilon}])}{\mu([\boldsymbol{\varepsilon}])} P(X \in[\boldsymbol{x}]),
$$

where $\mu$ is Borel measure on $\mathbb{R}^{p}$. This definition checks properties (6) and (7). It also tries a rough approximation for $P(\boldsymbol{X} \in[\boldsymbol{x}] \& g(\boldsymbol{X}) \in[\boldsymbol{\varepsilon}])$.

- The definition of Cut is an important choice. There are $n$ possible bisections of $[\boldsymbol{x}] \in\left[\mathbb{R}^{n}\right]$. Our algorithm selects a bisection $([\boldsymbol{y}],[\boldsymbol{z}])$ randomly in regards to the following criterions:

- Favor cuts such that $\omega_{[y]} \ll \omega_{[z]}$ or $\omega_{[y]} \gg \omega_{[z]}$,

- Avoid overly elongated $[\boldsymbol{x}]$, ie. $\frac{\max _{i}\left(x_{i}^{+}-x_{i}^{-}\right)}{\min _{i}\left(x_{i}^{+}-x_{i}^{-}\right)} \gg 1$,

In addition, this cut process interacts with some cuts history simplifications (next point).

- Our implementation tries to optimize the structure of the cuts history. For example, assume that $([\boldsymbol{y}],[\boldsymbol{z}])$ is a cut of $[\boldsymbol{x}],([\boldsymbol{t}],[\boldsymbol{u}])$ is a cut of $[\boldsymbol{z}]$ and $[g]([\boldsymbol{u}]) \cap[\boldsymbol{\varepsilon}]=\emptyset$, ie. $[\boldsymbol{u}]$ is outside the subpaving and its border. Then, boxes $[\boldsymbol{z}]$ and $[\boldsymbol{u}]$ should be 
removed from the structure, since they convey no useful information:

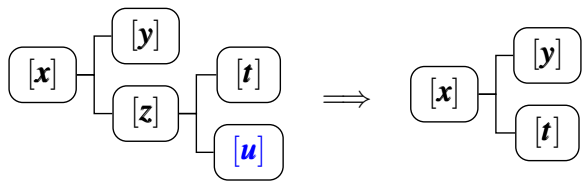

- $M$ first samples are discarded before sampling, so as to initialize the structure of the sampler. After that, $N$ samples are sampled and returned.

Whatever, the algorithm is rather simple to parameter. Except for the choice of $\omega$, which is structural, $r, M$ and $\sigma$ are the only parameters to be defined.

\subsection{Some ideas for improvements}

As will be shown in the tests, our method has been applied up to a space of dimension $n=11$ (for a subvariety of dimension $v=10$ ). This is much more than what is possible through a complete subpaving construction. But somehow, it is a reprieve in regards to the curse of the dimension.

Using contractors. Contractors are used along with bisection process in order to speedup the subpaving construction (Chabert and Jaulin, 2009). Contractors are especially available when function $g$ is expressed through some constraints. Such tool may be usefull in our algorithm, in terms of improving the speed and reducing the complexity of the cuts history.

Better weighting function. Definition (8) is rather cheap. Defining $\omega$ as a better approximations of $P(\boldsymbol{X} \in[\boldsymbol{x}] \& g(\boldsymbol{X}) \in[\boldsymbol{\varepsilon}])$ may be possible by means of local linear approximations (or highr order) of $g$.

Relaxing the constraint. Many rare event simulation methods work by starting from a relaxed constraint, e.g. $h(\boldsymbol{x}) \geq \gamma_{0}$ where $\gamma_{0} \ll \gamma_{\max }$, then by gradually tensing this constraint, e.g. up to $h(\boldsymbol{x}) \geq \gamma_{k}$ where $\gamma_{k} \simeq \gamma_{\max }$. Our algorithm totally discarded such approaches. However, it may be profitable to mix both points of view in order to improve the behavior of our approach for higher dimensions. We have especially in mind a way for an incrementally better definition of the weighting function $\omega$.

Parallelization. Our implementation is singlethreaded. But the process is clearly parallelizable by generating groups of samples in parallel. However, updates to cuts and omg need synchronization.

\section{EXAMPLES AND TESTS}

This section is parted in two subsections. First subsection presents simulation tests. Second section presents a small application in black-box function optimization. Such applications were actually a great motivation for this work.

\subsection{Simulation tests}

The tests presented here are performed for our sampling algorithm 3. The algorithm has been implemented in rust language (www.rust-lang.org) and, for now, is single-threaded. In order to illustrate the performance of this algorithm, our choice was to consider a mathematically simple simulation problem, in order to make the statistics of the results clear enough to analyze.

\subsubsection{Test cases}

Thorough the section, it is assumed that $\boldsymbol{X}$ follows the uniform law on $\boldsymbol{b}=[-2,2]^{n}$ with $n \in\{2,3, \cdots, 11\}$. Three simulation cases are investigated:

Case (a): Are defined $g_{a}(\boldsymbol{x})=\|\boldsymbol{x}\|_{2}=\sqrt{\sum_{j=1}^{n} x_{j}^{2}}$ and $\left[\boldsymbol{\varepsilon}_{a}\right]=[0.95,1.05]$. Then $g_{a}^{-1}\left(\left[\boldsymbol{\varepsilon}_{a}\right]\right)$ is a hyperannulus, which approximates the unit hypersphere of dimension $n-1$.

Case (b): For $n=11$ and $0 \leq k \leq 9$, it is considered:

$$
g_{b}(\boldsymbol{x})=\left[\begin{array}{c}
\|\boldsymbol{x}\|_{2} \\
\left|x_{3}\right| \\
\vdots \\
\left|x_{2+k}\right|
\end{array}\right] \text { and }\left[\boldsymbol{\varepsilon}_{b}\right]=\left[\boldsymbol{\varepsilon}_{a}\right] \times[\boldsymbol{z}]^{k},
$$

with $[z]=[-0.05,0.05]$. This case is similar to (a), but with additional constraints, so that $g_{b}^{-1}\left(\boldsymbol{\varepsilon}_{b}\right)$ approximates an hypersphere of dimension $n-1-k$. When $k=0$, there is no additional constraints and we are back to case (a) with $n=11$. When $k=9$, then $g_{b}^{-1}\left(\boldsymbol{\varepsilon}_{b}\right)$ approximates the unit circle $\mathcal{C}$ within the first two dimensions.

Case (c): For $n=11$, it is considered:

$$
g_{c}(\boldsymbol{x})=\left[\begin{array}{c}
|| \boldsymbol{x}||_{2} \\
\left|x_{3}\right| \\
\vdots \\
\left|x_{11}\right| \\
\min \left(\left|x_{1}\right|,\left|x_{2}\right|\right)
\end{array}\right],
$$

and $\left[\boldsymbol{\varepsilon}_{c}\right]=\left[\boldsymbol{\varepsilon}_{a}\right] \times[\boldsymbol{z}]^{9} \times[\boldsymbol{\alpha}]$ with $[\boldsymbol{\alpha}]=[-0.5,0.5]$. It is noticed that this case is obtained by adding contraint $-0.5 \leq \min \left(\left|x_{1}\right|,\left|x_{2}\right|\right) \leq 0.5$ to last subcase of (b). In other words, we are approximately sampling on the 
(disjoint) union of the 4 subsegments $\mathcal{A}_{1}, \ldots, \mathcal{A}_{4}$ of the unit circle $\mathcal{C}$, defined by:

$\mathcal{A}_{j}=\left\{\left[\begin{array}{l}x_{1} \\ x_{2}\end{array}\right] \in \mathcal{C} / \arg \left(\left[\begin{array}{l}x_{1} \\ x_{2}\end{array}\right]\right) \in j \frac{\pi}{2}+\left[-\frac{\pi}{6}, \frac{\pi}{6}\right] \bmod 2 \pi\right\}$.

These 4 subsegments have exactly the same size so that their (infinitesimal) probabilities are the same in regards to $X$.

Purpose of the test cases: Subsequently, case (a) is used in order to evaluate the performance of the sampling process both in accuracy and in efficiency for different dimensions. Case (b) is used in order to evaluate the efficiency of the sampling when the number of constaints increases. Case (c) is used in order to evaluate the accuracy of the sampling in case of complex constaints which introduce disjoint modalities.

\subsubsection{Results and analysis}

The cases considered here are mathematically easy to predict. Case (a) especially is rather easy to sample by ad hoc methods. In (Dezert and Musso, 2001), Dezert and Musso proposed a method, which may be used for uniformly sampling on an annulus of ellipsoid. But the problem is not trivial however, since one have to take into account the variation of the local probability with the radius. Whatever, one must keep in mind that our approach is generic and can be applied to an infinite number of configurations.

All the subsequent tests have been achieved with the following parameters:

- $r=0.002$ is the radius bound for second while stop condition,

- $M=5000$ is the number of first samples, which are discarded in order to initialize the sampler,

- $N=50000$ is the number of sampled particles.

- $\sigma=10$ on all tests.

\section{Case (a):}

Histograms: For each subcase $n \in\{3,7,11\}$, we have computed the radius of all samples $x$ and built the associated histograms (figures 2, 3 and 4).

For each $n \in\{3,7,11\}$ and for all $1 \leq i<j \leq n$, we have computed the angle of all samples $\left(x_{i}, x_{j}\right)$ and built the associated histograms. From these $\frac{n(n-1)}{2}$ histograms of each subcase, we have computed the minimal, mean and maximal histogram. By symmetry, the theoretical histograms are uniform.

The results are shown respectively in blue, green and

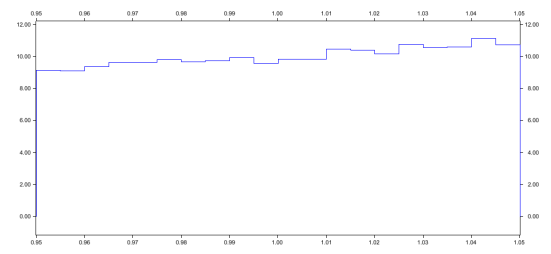

Figure 2: (a):Radius histogram (20 divisions); $n=3$

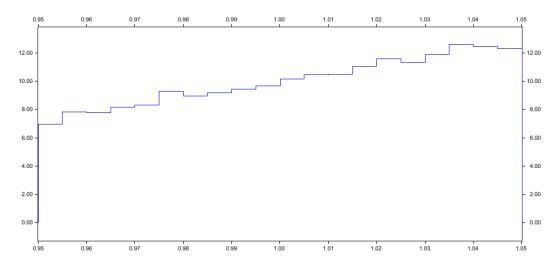

Figure 3: (a): Radius histogram (20 divisions); $n=7$

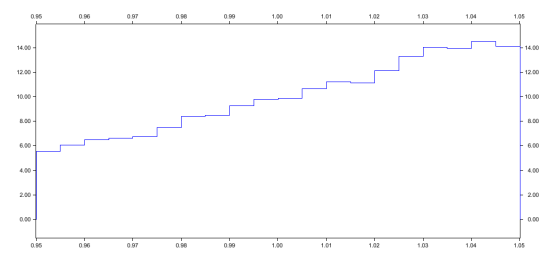

Figure 4: (a): Radius histogram (20 divisions); $n=11$

red (figures 5, 6 and 7), and provide an hint on the error of the estimation.

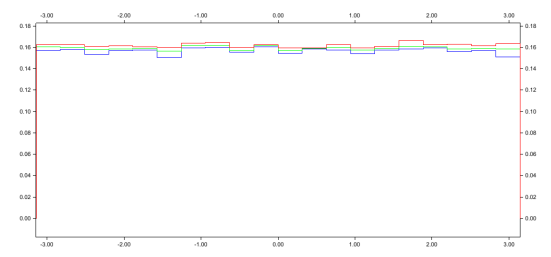

Figure 5: (a):Angle histogram (20 divisions); $n=3$

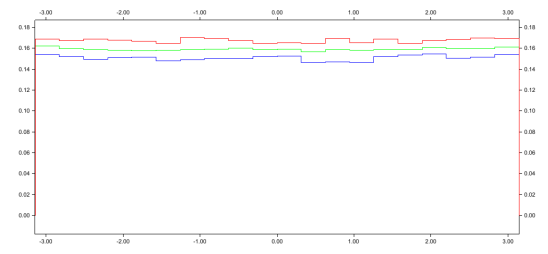

Figure 6: (a): Angle histogram (20 divisions); $n=7$

The error on the histograms should be of the order of $\sqrt{\frac{20}{50000}} \simeq 0,02$. In comparaison, the errors figured in the angular histograms are quite acceptable, even for the highest dimension. The most interesting point is that there is no rupture in the histogram, which shows that the sampler does manage the subvariety structure. 


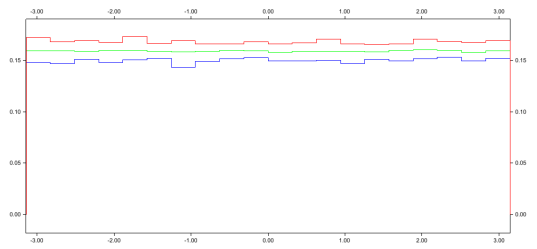

Figure 7: (a): Angle histogram (20 divisions); $n=11$

We do not have an error estimation for the radius histograms. Actually, the local probability should theoretically increase with the radius, this property being accentuated with the dimension. This is what is obtained on the histograms. It is noteworthy however that the sides of these histogram are subject to additional errors implied by the border of the subvariety.

Process statistics: We present some elements of measurement on the behavior of the algorithm according to the number of generated samples (0 to 55000). Each figure presents the result for cases $n=3,7,11$ with respective colors, blue, green and red.

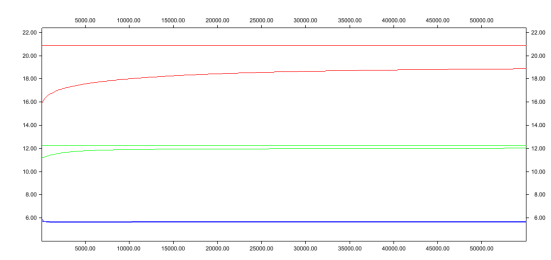

Figure 8: (a): omg $([\boldsymbol{b}])$ versus $P\left(g_{a}(\boldsymbol{X}) \in[\boldsymbol{\varepsilon}]\right) ; n=3,7,11$

Figure 8 presents how value $\log _{2}($ omg $([\boldsymbol{b}]))$ evolves as an approximation of $\log _{2}\left(P\left(g_{a}(\boldsymbol{X}) \in[\boldsymbol{\varepsilon}]\right)\right)$. While all curves are increasing to this theoretical value (line of the same color), the performance decreases with the dimension.

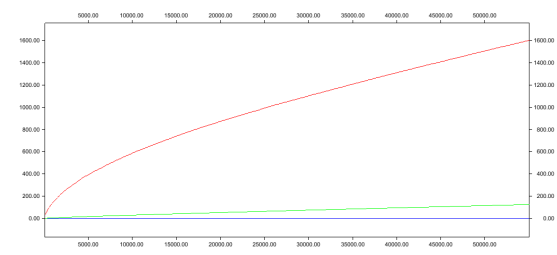

Figure 9: (a): Cumulative cpu time; $n=3,7,11$

Figures 9 and 10 present the cumulative cpu-time (expressed in second) and the evolution of the cpu-time (per sample) consumed by the process. We notice clearly that the sampling efficiency increases with the number of generated samples. However, the cumulative cpu time still increases dramatically with the dimension (the memory use evolves similarly). Although the curse of dimension has been delayed by

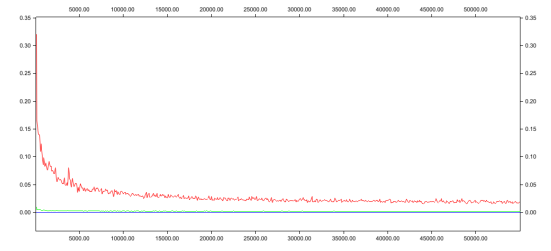

Figure 10: (a): Evolution of cpu time; $n=3,7,11$

our approach, it is still there.

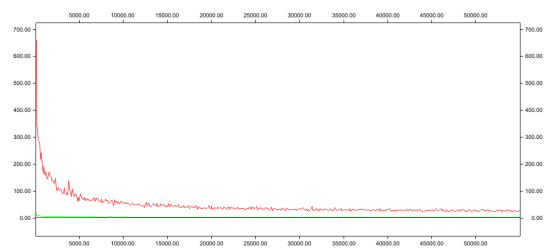

Figure 11: (a): Evolution of loop retry; $n=3,7,11$

The number of loop retries during the sampling is an interesting indication of the achievement of the sampling structure (figure 11). It decreases with the number of samples, and moreover becomes rather small, even for the highest dimension (around 30 for $n=11$ ). This result should be compared the probability of the subvariety (around $10^{-6}$ for $n=11$ ).

Case b: We present now some synthetic curves on the sampler efficiency, when the number of constraints increases. All curves are function of $k$, the number of additional constraints. The values are computed as the mean on the 100 last samples (thus, from 49901 to 50000).

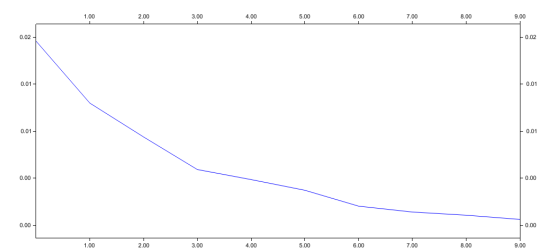

Figure 12: (b): Evolution of cpu time; $k=0: 9$

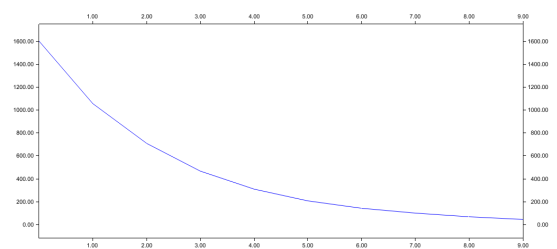

Figure 13: (b): Evolution of cumulative cpu time; $k=0: 9$

Figures 12 and 13 present the evolution of respec- 
tively the cpu time (per sample) and cumulative cpu time in function of $k$. The curves are decreasing and are nearing to zero. Figure 14 presents the evolution

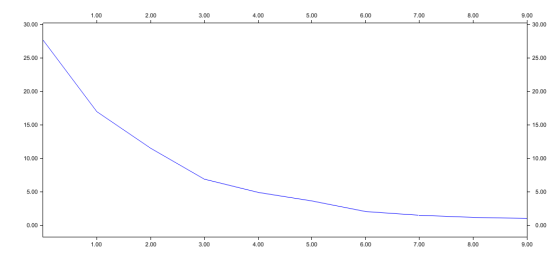

Figure 14: (b): Evolution of loop retry; $k=0: 9$

of the number of loop retries in function of $k$. Again, the curve is decreasing and is nearing to zero.

As a conclusion here, the performance of the sampler tends to increase with the number of constraints, and this is a quite useful quality.

Case c: On this last test, we computed the radial histogram and angular histogram (there is only one) for the samples. These histograms are presented respectively in figures 15 and 16 . The quality of the histograms is comparable to what have been seen in the previous cases. Due to the constraint configuration, the theoretical radius histogram is uniform, and the theoretical angular histogram is uniform around each subsegment, $\mathcal{A}_{1}, \ldots, \mathcal{A}_{4}$ with a gradual decrease on the borders. The generated histograms actually comply with these properties.

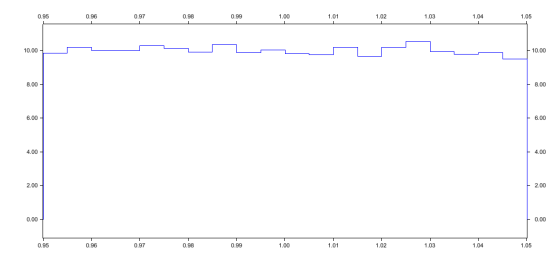

Figure 15: (c): Radius histogram (20 divisions)

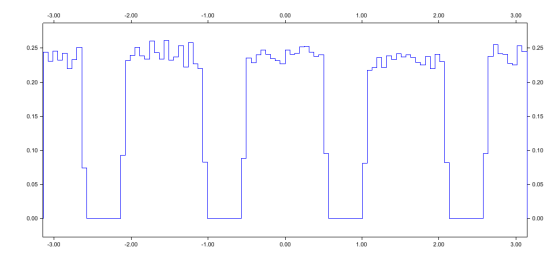

Figure 16: (c): Angle histogram (100 divisions)

As a preliminary conclusion, we consider that our sampling method is globally performant in sampling conditionally to subvarieties. A future issue will be to increase the dimension of the sampling space.

\subsection{Optimizing a black-box function}

Assume that one needs to optimize a function which is not well known, and which may be computed by a highly costy process (a heavy simulation, tests made by human teams on the grounds, etc.). Of course the optimization should be made by sparing at best the number of calls to the costy evaluation. Is it possible to solve that? At first sight, it is tempting to say no!

In (Jones et al., 1998), Jones, Schonlau and Welch proposed an elegent method (EGO) for addressing such kind of problem. The idea is to use a surrogate function under the form of a functional random variable. This functional random variable is described by means of a Gaussian process with correlation depending on spatial distance (kriging). Based on such modelling, the construction of an optimal parameter sequence to be evaluated is obtained by iterating:

- Compute the posterior law of the functional random variable, according to the past evaluations,

- Compute the expected improvement function, in regards to the posterior law and the already best computed value,

- Choose the parameter optimizing this expected improvement function and evaluate it.

It is beyond the scope of this paper to detail this seminal work, but we describe subsequently a non-linear reinterpretation of the method. Whatever, the approach relies on deep simplifications obtained from the Gaussian modelling, and it tends to be less efficient when the dimension of the optimization space increases. Works have been made in order to deal with this dimentional issue; eg. (Bouhlel et al., 2016).

\subsubsection{A non-linear formalisation}

We intend to solve this simple geometric problem: how to find the isobarycenter $\gamma=(a, b) \in[-5,5]^{2}$ of 4 unknown points $M_{i}=\left(x_{i}^{o}, y_{i}^{o}\right) \in[-5,5]^{2}$ with $i \in\{1,4\}$ ? The only approach that is possible for us is to test some solutions by requesting for a costly measurement; this measurement evaluates function:

$$
g\left(\boldsymbol{\gamma}, \boldsymbol{x}^{o}\right)=\left\|\gamma-h\left(\boldsymbol{x}^{o}\right)\right\|_{2} \text { with } h\left(\boldsymbol{x}^{o}\right)=\frac{1}{4} \sum_{i=1}^{4} M_{i},
$$

where $\boldsymbol{x}^{o}=\left(x_{1}^{o}, y_{1}^{o}, \ldots, x_{4}^{o}, y_{4}^{o}\right)$. Our purpose is then to optimize $(a, b)$ by doing a minimum request to the costly evaluation $g\left(\gamma, x^{o}\right)$

In (Dambreville, 2015), we proposed a reinterpretation of EGO by considering the non-linear function $g(\boldsymbol{\gamma}, \boldsymbol{X})$ depending on noise model $\boldsymbol{X}$ instead of a kriging-based surrogate function. Then, the optimization of measure sequence $\left(\gamma_{k}\right)$ is obtained by iterating: 


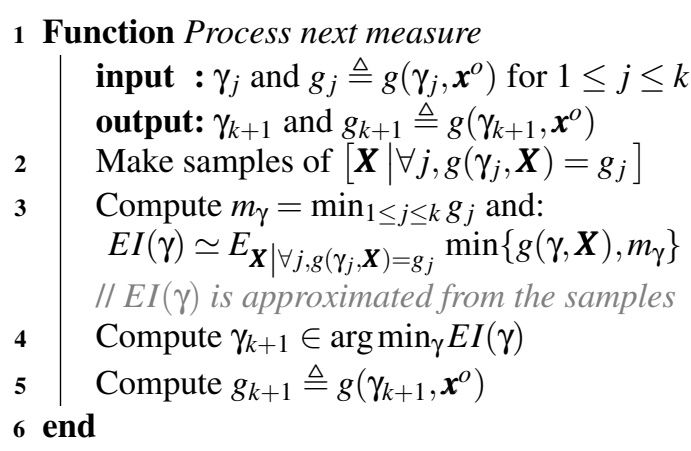

We did not have an efficient method for sampling $\left[\boldsymbol{X} \mid \forall j, g\left(\gamma_{j}, \boldsymbol{X}\right)=g_{j}\right]$ at the time of (Dambreville, 2015). Now, we propose to apply algorithm 3 combined with a discretized method (we actually enumerated on $10^{6}$ discretized points of $[-5,5]^{2}$ ) for minimizing the expected improvement $E I(\gamma)$ in order to process minimizing sequence $\left(\gamma_{k}\right)$. This sequence converge to an isobarycenter for $M_{1: 4}$.

\subsubsection{Tests and results}

The points are $M_{1: 4}=(2,-1),(3,2),\left(-\frac{3}{2}, 4\right),\left(\frac{1}{2}, 3\right)$. Their barycenter is $(1,2)$. We used a sampler with $M=500, N=1000$ and $[\boldsymbol{\varepsilon}]=\left[-\frac{1}{100}, \frac{1}{100}\right]^{k}$. Variable $\boldsymbol{X}$ is considered uniform on $[-5,5]^{8}$. The optimization is done on 13 evaluations. If the optimum is obtained at step $k_{o}$, then the process is stopped. The following table summarizes the results of 100 runs. $m_{o}$ and $\gamma_{o}$ are respectively the best computed evaluation and solution. cpu is the mean computation time:

\begin{tabular}{|c||c|c|c|c|c|c|}
\hline$k_{o}$ & 3 & 4 & 5 & 9 & 10 & 13 \\
\hline$\%$ & 14 & 52 & 31 & 1 & 1 & 1 \\
\hline$m_{o}$ & 0 & 0 & 0 & 0 & 0 & 0.01 \\
\hline$\gamma_{o}$ & $(1,2)$ & $(1,2)$ & $(1,2)$ & $(1,2)$ & $(1,2)$ & $(0.99,2)$ \\
\hline $\mathrm{cpu}(s)$ & 973 & 125 & 42 & 62 & 67 & 84 \\
\hline
\end{tabular}

Barycenter is mainly found after 3 to 5 evaluations. This is close to the geometric method, which gives the solution equiprobably after 3 or 4 evaluations. ${ }^{4}$

\section{CONCLUSIONS}

We proposed an original dichotomous method for sampling a random vector conditionnaly to a subvariety. This generic approach, inspired from interval analysis, is accurate and efficient up to a space of dimension 11. We have shown how it could be applied efficiently to the optimization of expensive black-box

\footnotetext{
${ }^{4}$ Each measure restricts the solution to a circle. After 2 measures, we usualy have to choose between two points, and the solution is found at step 3 or 4 .
}

function. The work is promizing from an applicative point of view and offers several improvement perspectives. We will particullarly investigate some parallelization issues and relaxations techniques applied to the subvariety in order to enhance the efficency of the approach in regards to higher dimensions.

\section{REFERENCES}

Abdallah, F., Gning, A., and Bonnifait, P. (2008). Box particle filtering for nonlinear state estimation using interval analysis. Automatica, 44(3):807-815.

Alefeld, G. and Mayer, G. (2000). Interval analysis: theory and applications. Journal of Computational and Applied Mathematics, 121(1):421-464.

Bouhlel, M., Bartoli, N., Morlier, J., and Otsmane, A. (2016). Improving kriging surrogates of highdimensional design models by partial least squares dimension reduction. Structural and Multidisciplinary Optimization, 53:935-.

Bui Quang, P., Musso, C., and Le Gland, F. (2016). Particle filtering and the Laplace method for target tracking. IEEE Transactions on Aerospace and Electronic Systems, 52(1):350-366.

Cérou, F., Del Moral, P., Furon, T., and Guyader, A. (2012). Sequential Monte Carlo for rare event estimation. Statistics and Computing, 22(3):795-908.

Chabert, G. and Jaulin, L. (2009). Contractor Programming. Artificial Intelligence, 173:1079-1100.

Dambreville, F. (2015). Optimizing a sensor deployment with network constraints computable by costly requests. In Thi, H. A. L., Dinh, T. P., and Nguyen, N. T., editors, Modelling, Computation and Optimization in Information Systems and Management Sciences, volume 360 of Advances in Intelligent Systems and Computing, pages 247-259. Springer.

Dezert, J. and Musso, C. (2001). An efficient method for generating points uniformly distributed in hyperellipsoids. In Workshop on Estimation, Tracking and Fusion: A Tribute to Bar-Shalom, Monterey, California.

Jaulin, L., Kieffer, M., Didrit, O., and Walter, E. (2001). Applied Interval Analysis with Examples in Parameter and State Estimation, Robust Control and Robotics. Springer London Ltd.

Jones, D., Schonlau, M., and Welch, W. (1998). Efficient global optimization of expensive black-box functions. Journal of Global Optimization, 13:455-492.

Morio, J., Balesdent, M., Jacquemart, D., and Vergé, C. (2014). A survey of rare event simulation methods for static input-output models. Simulation Modelling Practice and Theory, 49:287-304.

Musso, C., Champagnat, F., and Rabaste, O. (2016). Improvement of the laplace-based particle filter for trackbefore-detect. In 2016 19th International Conference on Information Fusion (FUSION), pages 1095-1102.

Rubinstein, R. Y. and Kroese, D. P. (2004). The Cross Entropy Method: A Unified Approach To Combinatorial Optimization, Monte-Carlo Simulation (Information Science and Statistics). Springer-Verlag. 